\title{
Development of special software for disc brake design
}

\author{
Jian Zhang \\ College of mechanical engineering \\ Jiangsu University of Science and Technology \\ Zhenjiang (212013), China \\ Zhjian127@163.com \\ Ben Zhang* \\ College of mechanical engineering \\ Jiangsu University of Science and Technology \\ Zhenjiang (212013), China \\ Zhangben2214@163.com \\ * Corresponding author
}

\author{
Yongmei Zhu \\ College of mechanical engineering \\ Jiangsu University of Science and Technology \\ Zhenjiang (212013), China \\ zymtt@163.com \\ Wenxian Tang \\ College of mechanical engineering \\ Jiangsu University of Science and Technology \\ Zhenjiang (212013), China \\ tangwenxian@163.com \\ Xilu Zhao \\ College of mechanical engineering \\ Saitama Institute of Technology \\ Fukaya, Japan \\ Zhaoxilu@sit.ac.jp
}

\begin{abstract}
Special software for disc brake was developed based on Visual Basic language, in order to design the brake automatically and professionally. The developing principles were easy application, professional design and intelligent operation. Calculating and checking patterns for the main parts of the brake were presented using programming languages. Once the design parameters were input into the software, structural and checking information of the brake components was proposed, which could provide references for the designer. The design and checking report was output with word format, when the analysis data met requirements. The results indicate that the software is easy and efficient to operate.
\end{abstract}

Keywords- disc brake; design software development; Visual Basic language; check; calculate

\section{INTRODUCTION}

Automobile is one of the most important and most convenient vehicles in the modem world. Brake system is an important part on the automobile chassis, it is a device to restrict automobile movement. Brake is the most important security component in the vehicle and greatly contributes to the performance of the vehicle ${ }^{[1]}$. Braking ability affects the security of the automobile. People's demand for safety and reliability is getting higher, with the highway speed and traffic density increasing. The automobile should be equipped with reliable brake system so that it could guarantee the safety of personal and automobile ${ }^{[2]}$. So it is important to complete the design of the brakes and the security correction.

Nowadays, people usually use expert system to design brake. In the overall design of the brake, we can use expert system to distribute braking force and chose the model of the brake ${ }^{[3]}$. The expert system can call the parameterized graphics library to select the component model, then it would call the CAD generates 3D entity model or twodimensional engineering drawing automatically in the detailed design ${ }^{4]}$. However, there is a lack of a professional platform on the parameter design, checking and generating design specification of the main parts of a disc brake ${ }^{[5]}$.

For this problem, this article exploits disc brake design system by stereotyping the process of disc brake design and check, realizing disc brake parametric design, integration check, generating document automatically and so on.

\section{DESIGN AND IMPLEMENTATION OF DISC BRAKE DESIGN SYSTEM}

The brake design and checking processes involve complex calculations, so we need recalculate and recheck when any parameter is changing. These repetitive behaviors are inefficient and costing a lot of effort and time.

The purposes of the design of disk brake design system in this article are:

(1) To realize the modeled and parameterization in the design of disc brake.

(2) To realize the checking automatically about the results of the design and providing the accurate reference data for designer.

(3) To realize the extracting and processing the results automatically, then, search the template file to replace the corresponding parameters to generate design specification.

The overall structure of the system

The overall design scheme of this disc brake design system is displayed in figure 1.This system is composed of template file parameterized module, design and checking module and document generation module. The design of 
the disc brake is modeled and the parameterization is completed by some corresponding program. When the designer inputs relevant parameters, the program will extract the parameters to do some calculation and check the accuracy of the result. If the result of checking is not meeting the demands of design, it will be required to input relevant parameters again. If the result is meeting the requirement of design, it will be asked to do the next step. The program will search the template files to replace the corresponding parameters to generate particular design specification.

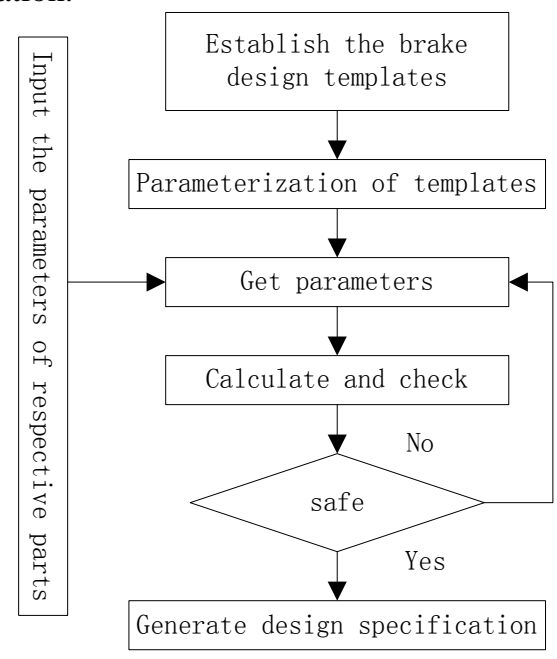

Figure 1. The overall design scheme of this disk brake design system.

The design and implementation of template file parameterized module

The overall design scheme of template file parameterized is shown in figure 2 .

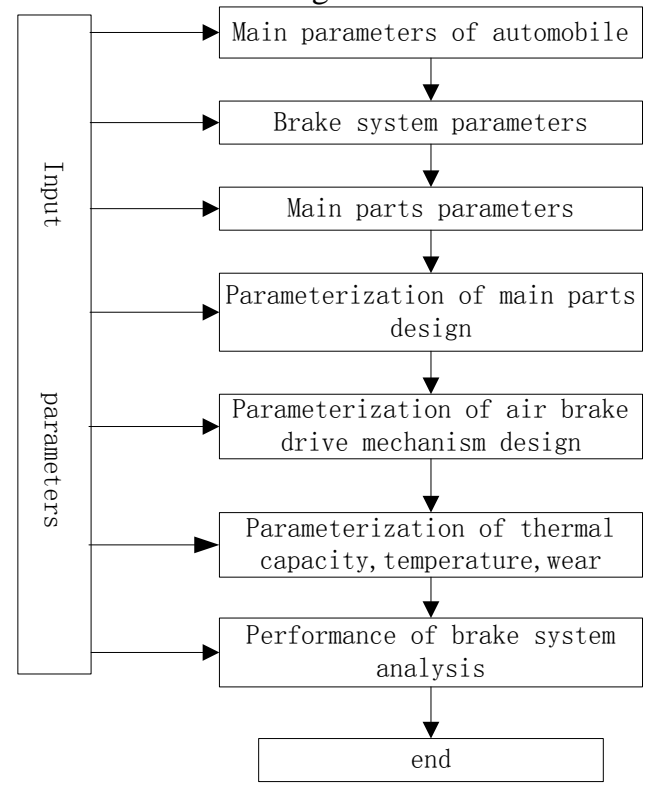

Figure 2. The overall design scheme of template file parameterized.

The design process about the disc brake is to be modeled and need to generate detailed design specification. Extract the relevant parameters which are needed in calculation and checking. The design and check which are needed in the process of the excogitation in this system are modeled by some programs. It is using unknown variables to indicate these parameters.

The main code is following:

......

mal $=$ TextBox1.Text 'the assignment of the main

$\operatorname{mq} 1=$ TextBox 2. Text parameters

mh1 $=$ TextBox3.Text

$\mathrm{Ful}=\varphi^{*} \operatorname{mal} *(\mathrm{~h} 1+\varphi * \mathrm{hg} 1) / \mathrm{L}$ 'calculation

$\mathrm{Fu} 2=\varphi^{*}$ ma1 - Fu1 modeling

$\beta r=F u 2 /(F u 1+F u 2)$

Fh1 = Math.Sqrt(Math.Pow $(\mathrm{Fq} 1,2)+\operatorname{Math} . \operatorname{Pow}(\mathrm{Ft} 1,2)$ $-2 * \mathrm{Fq} 1 * \mathrm{Ft} 1 * \operatorname{Math} . \mathrm{Cos}(103.6))$

.....

When designers input relevant designing parameters with the software of man-machine interface, the program will extract the parameters and then output the result of calculation automatically.

The design and implementation of designing and checking module

The designing and checking module is concluding the main components design of the brake, the design of lever, stand and air chamber; the choice of bracket mounting bolt and pillars; the checking of warming and brake performance of disc brake, and so on. As is shown in figure 3 the system main interface.

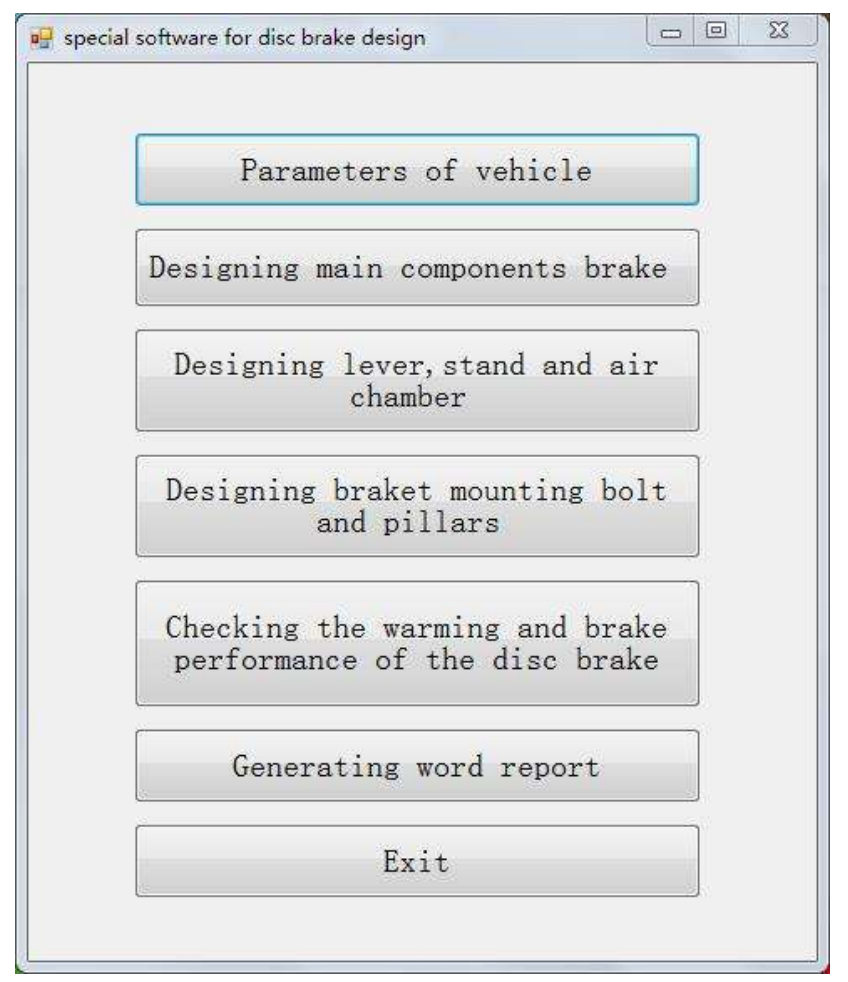

Figure 3. The system main interface 


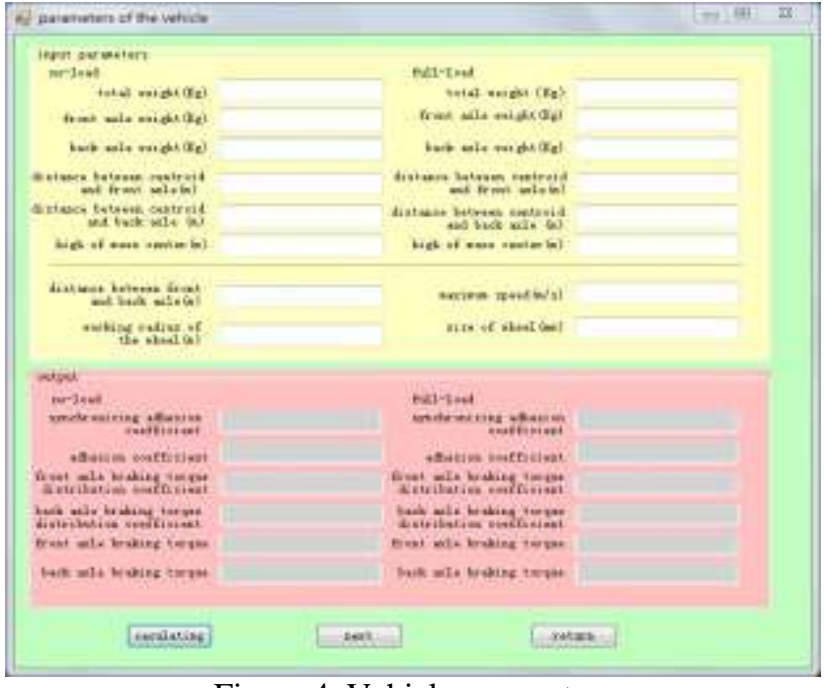

Figure 4. Vehicle parameters

Input the needed parameters through the vehicle parameters interface displayed in figure 4.The system will extract the parameters and assign to corresponding unknown variables, then do some calculation and finally output the results.

Input relevant parameters through the major parts of the brake design interface which is shown in figure 5 .

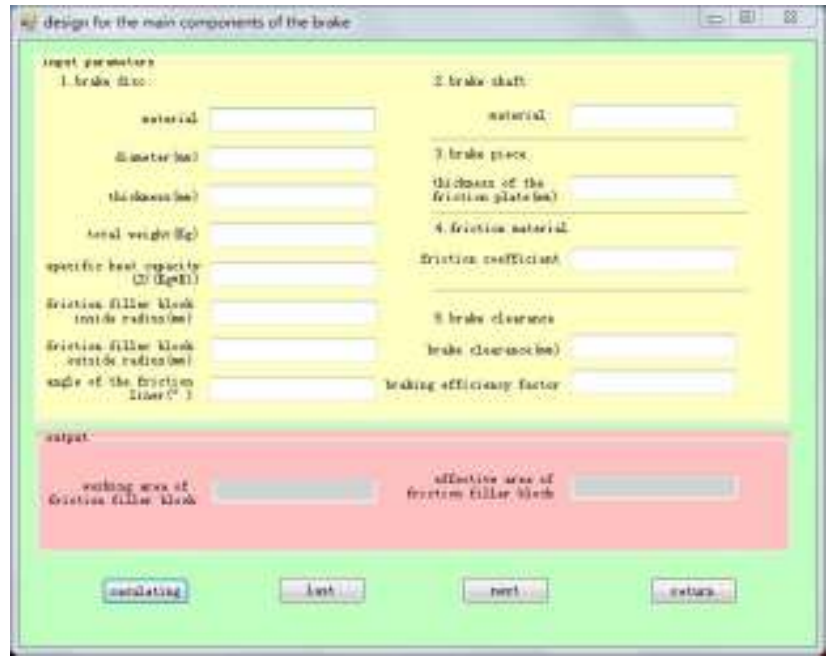

Figure 5. Major parts of the brake design

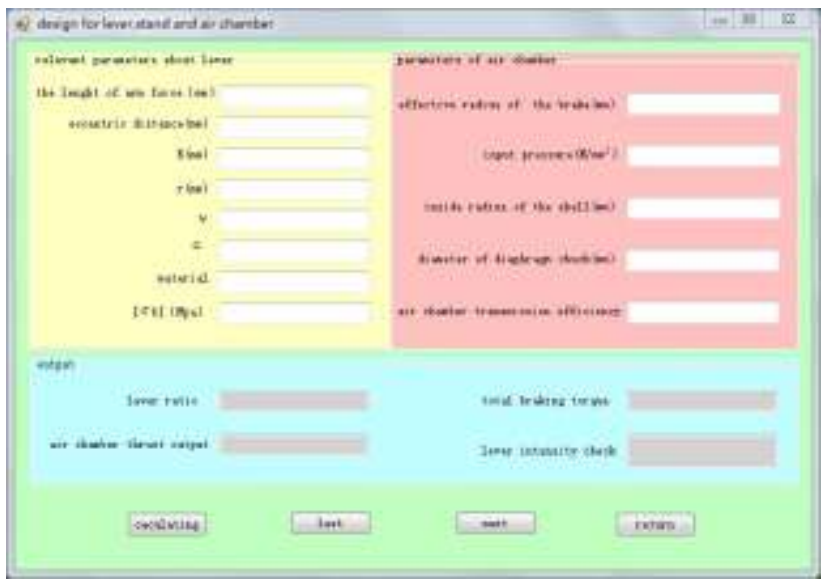

Figure 6. lever, stand and air chamber design

The system will extract the parameters and do some calculation, then output results.

Input relevant parameters through the lever, stand and air chamber design interface in figure 6 . The system will extract parameters to calculate, then it will check the result and display the consequence. If the results meet the requirements, it will do the next step. Otherwise it will reenter the parameters and check the results again. It will not stop until the results meet the design requirements.

Input relevant parameters through the stand erection bolt and guide pin choose interface in figure 7 .

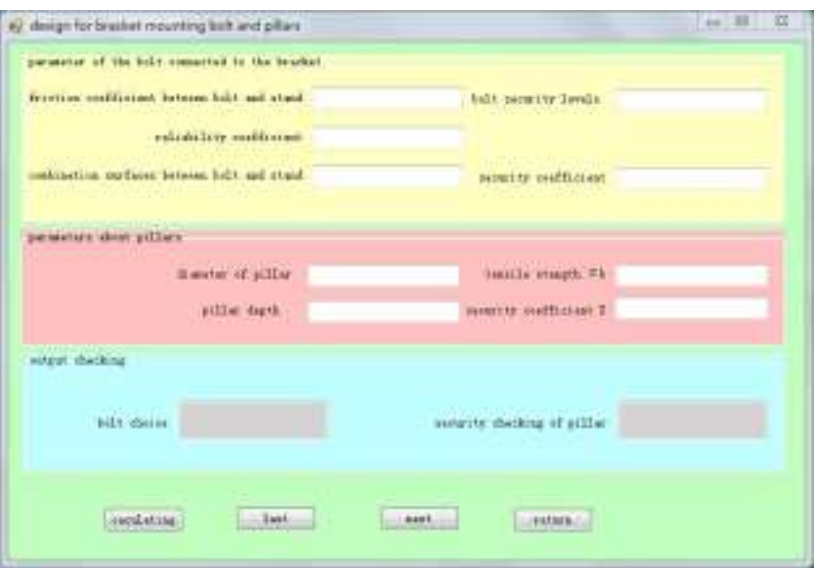

Figure 7. Stand erection and guide pin choose

The system will extract parameters to calculate, and compare results with the standard size. It will choose the right bolt that complied with the design requirement. After that, it will check the safety factor of guide pin and display the check result. If the results meet the requirements, it will do the next step. Otherwise it will re-enter the parameters and check the results again. It will not stop until the results meet the design requirements.

Input relevant parameters through the brake warming and performance check interface in figure 8 .

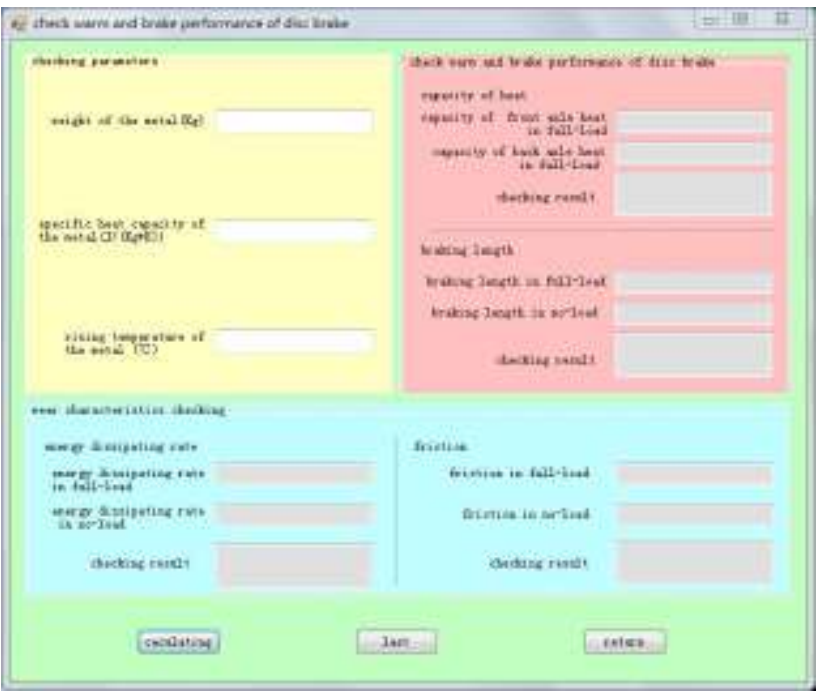

Figure 8. Brake warming and brake performance check 
The system will input relevant parameters and check warming and performance of the brake on the bases of the former results. If the results meet the requirements, it will do the next step. Otherwise it will re-enter the parameters and check the results again. It will not stop until the results meet the design requirements.

The main code is following:

$$
\text { jsd }=1.8 *(\text { Mu1max }+ \text { Mu2max }) /(\operatorname{Re} * \operatorname{ma} 2)
$$$$
\mathrm{t} 2=18 / \mathrm{jsd}
$$$$
\mathrm{e} 2=\mathrm{ma} 2 * 324 * \beta /(4 * \mathrm{t} 2 * \mathrm{Am})
$$$$
\mathrm{e} 3=\mathrm{ma} 2 * 324 *(1-\beta) /(4 * \mathrm{t} 2 * \mathrm{Am})
$$$$
\mathrm{jsdk}=1.8 *(\operatorname{Mu} 1 \operatorname{maxk}+\mathrm{Mu} 2 \mathrm{maxk}) /(\operatorname{Re} * \operatorname{ma} 1)
$$$$
\text { .... }
$$$$
\mathrm{e} 2 \mathrm{k}=\text { Math.Round }(\mathrm{e} 2 \mathrm{k}, 3)
$$$$
\mathrm{e} 3 \mathrm{k}=\text { Math.Round }(\mathrm{e} 3 \mathrm{k}, 3)
$$

TextBox10.Text $=$ Math.Round(f0, 3) 'result output

TextBox11.Text $=$ Math.Round(f0k, 3)

If Math.Max(e2, e3) $<=6$ AndAlso Math.Max(e2k, e3k) $<=6$ Then 'check

TextBox9.Text $=$ "dissipative rate of energy is less than $6 \mathrm{w} / \mathrm{mm}^{2}$, cut the mustard "

ElseIf Math.Max(e2, e3) > 6 Then

TextBox9.Text $="$ full load dissipation rating is greater than $6 \mathrm{w} / \mathrm{mm}^{2}$, don't meet the requirement "

Else : TextBox9.Text $=$ " no- load dissipation rating is greater than $6 \mathrm{w} / \mathrm{mm}^{2}$, don't meet the requirement "

End If

If f0 $<=2$ AndAlso f0k $<=2$ Then

TextBox12.Text $=$ "friction force is less than $2 \mathrm{~N} / \mathrm{mm}^{2}$, cut the mustard"

ElseIf $\mathrm{fO}>2$ Then

TextBox12.Text = "full load friction is less than $2 \mathrm{~N} / \mathrm{mm}^{2}$, don't meet the requirement"

Else : TextBox12.Text $=$ "no-load friction is less than $2 \mathrm{~N} / \mathrm{mm}^{2}$, don't meet the requirement "

End If

If $\mathrm{Sjl}<=21$ AndAlso Sjlk $<=20$ Then

TextBox6.Text $=$ "brake performance is meet the requirement "

ElseIf Sjl > 21 Then

TextBox6.Text = "full load braking length greater than $21 \mathrm{~m}$, don't meet the requirement "

Else : TextBox6.Text = "no-load braking length greater than $21 \mathrm{~m}$, don't meet the requirement "

End If

If Lrn1 <= Lrn AndAlso Lrn $2<=$ Lrn Then

TextBox3.Text $=$ "calorific capacity is meet the requirement "

ElseIf Lrn1 > Lrn Then

TextBox3.Text $=$ "full load calorific greater than " \& Lrn \& "don't meet the requirement "

Else : TextBox3.Text $=$ " no-load calorific greater than " \& Lrn \& " don't meet the requirement "

End If

The design and implementation of document generation module

VB.NET can call Word to output report by add COM port. It can stick labels on the parameters which involved in the process of the design. The system will search the labels in the word template and replace them with the results of calculation and check. Then it will generate detailed design specifications.

The main code is following:

.....

yourform.Label1.Font = New Font("song typeface", 15) yourform.Label1.Text $=$ ("being generate word document, please wait .....") ' replace the data with the output parameters

filename = "e:\1.docx"

mWordapp $=$ CreateObject $($ "Word.Application")

mobjDoc $=$ mWordapp.Documents.Add(filename, missing, missing, missing)

mWordapp. Visible $=$ False

mWordapp.ActiveDocument.Bookmarks.Item("ma1").

Select()

mWordapp.Selection.TypeText(ma1)

.....

'save the generated word document then quit.

mobjDoc.SaveAs("c:lword document.doc")

mWordapp.Application.Quit(missing, missing, missing) yourform.Hide()

MessageBox.Show("WORD document have been generated and saved on disc $\mathrm{C}$, the name of the document is 'word document' ", "hint", MessageBoxButtons.OK)

mWordapp $=$ Nothing

mobjDoc $=$ Nothing

.....

\section{CONCLUSIONS}

(1) The special software development for disc brake design is verified by the design of 24.5 inch disc brake. The results have revealed that the system is right and practical. So we can draw the following conclusions:

(2) The special software development for disc brake design modeled the process of brake design. It also reduced the difficulties in the design process and the load of the designers. It greatly improved working efficiency.

\section{REFERENCES}

[1] Jens Wahlstrom, Lars Olander, Ulf OlofssonNoble. Size,Shape,and Elemental Composition of Airborne Wear Particles from Disc Brake Materials. Tribology Letters,2013,Vol.38(1),pp.15-24

[2] Pyung Hwang, Xuan Wu, Investigation of temperature and thermal stress in ventilate disc brake based on $3 \mathrm{D}$ thermomechanical coupling model. Journal of Mechanical and Technology,2010,Vol.24(1),pp.81-84

[3] P.J.Blau, J.C.McLaughlin. Effects of water films and sliding speed on the frictional behavior of truck disc brake materials. Thribology International, 2013,Vol.36(10), pp.709-715

[4] Y.-G.Joe,B.-G.Cha,H.-J.Sim,H.-J.Lee,J.-E.Oh. Analysis of disc brake instability due to friction-induced vibration using a distributed parameter model. International Journal of Automotive Technology,2012,Vol.9(2)

[5] S.Qiao,D.M.Beloiu,R.A.Lbrahim. Deterministic and Stochastic Characterization of Friction-Induced Vibration in Disc Brakes .Nonlinear Dynamics,2012,Vol.36(2)

[6] Omar Maluf, Je.ferson Aparecido Moreto, Mauricio Angeloni. Thermonechanical and Isothermal Fatigue Behavior of Gray Cast Iron for Automotive Brake Discs. INTECH OPEN Access Publisher ,2011.

[7] Dragos B. Maciuca . Brake modelling and control . Intelligent Vehicle Technologies . Elsevier Inc . 2010

[8] Z. H . Yao, M. W . Yuan. Computational Mechanics .Springer Berlin Heidelberg . 2011 
[9] Jiusheng Bao, Min ming Tong, Zhencai Zhu. Intelligent Tribological Forecasting Model and System for Disc Brake. The $24^{\text {th }}$ Chinese Control and Decision Conference ,2011

[10] XIAO Cheng-yong, JIANG Yong ,HAO Zhi-jun. Analysis on Wet Multi-Disc Brake Noise Based on ABAQUS. $20122^{\text {nd }}$ International Conference on Machatronics and Automation (ICMMA 2012)
[11] Catalin Spulber, Stefan Voloaca . Aspects regarding the disc brake's thermal stress simulation by using Infrared Thermography. International Conference on Optimization of the Robots and Manipulators (OPTIROB 2011) 